fit for purpose in examining the epidemiology of specific conditions and contributing to planning decisions. Public health needs to be proactive in providing relevant needs assessment information to ensure that planning within financial constraints is appropriately informed by population needs.

\section{P1-374 QUALITY OF LIFE OF ADULTS AND ASSOCIATED FACTORS, BRAZIL}

doi:10.1136/jech.2011.142976f.66

${ }^{1} \mathrm{~F}$ Floriano, ${ }^{*} \mathrm{M}$ da C Costa, ${ }^{2} \mathrm{M}$ I Vianna, ${ }^{2} \mathrm{M}$ B Cabral, ${ }^{1} \mathrm{G}$ Santos, ${ }^{2} \mathrm{M}$ dos Santos, ${ }^{3} \mathrm{M}$ R Santos. ${ }^{1}$ ISC-UFBA, SSA, Bahia, Brazil; ${ }^{2}$ FO-UFBA, SSA, Bahia, Brazil; ${ }^{3}$ UCSAL, SSA, Bahia, Brazil

Introduction The objective of this study was to assess quality of life (QoL) among adults in Salvador-Bahia, Brazil, and identify potentials factors related to its domains.

Methods Cross-sectional study in 501 adults between 35 and 59 years, living in five different areas covered by the Community Health Agents Program/Family Health Strategy (CHAP/FHS), from July to October, 2008. Data were collected through interview using a questionnaire including social-economic variables and oral health status and the WHO Quality of Life questionnaire (WHOQOL-Bref) validated in Brazilian population to measured QoL. Descriptive and exploratory analyses were carried out and differences in scores were tested by Mann-Whitney and Kruskal-Wallis tests.

Results Mean scores of WHOQOL-Bref were $58.5 \pm 19.8$ and $59.0 \pm 27.3$ to the general issues of quality of life and satisfaction with health, respectively. The social relationships domain had the highest mean scores $(69.3 \pm 18.4)$ and the environment domain had the lowest mean scores $(49.8 \pm 14.5)$. The WHOQOL-Bref domains showed that $\mathrm{QoL}$ is perceived better by male, having the highest mean scores $(71.8 \pm 12.8)$ on the psychological domain and the lowest mean scores $(53.8 \pm 13.1)$ on the environmental domain. Variables predicted for QoL were: years of education, monthly individual income, treated water at home and mucosal damage.

Conclusions Evaluate QoL is very important for adult and it showed associated with current social-economic conditions and with oral health status. WHOQoL-Bref proved to be an efficient tool and useful for a better evaluation approach of the subjective aspects of QoL.

\section{P1-375 MELANOMA IN SWITZERLAND: AN UNMET NEED FOR PREVENTION?}

doi:10.1136/jech.2011.142976f.67

N Wyss, ${ }^{*}$ E Roy. Office fédéral de la statistique, Neuchâtel, Switzerland

Introduction Cutaneous malignant melanoma incidence rate has been increasing in Caucasian populations in the last decades. This trend is also observed in Switzerland where one of the highest incidence rates in the world is registered. This paper examines time trends in incidence, mortality and prevention practices in Switzerland over the last decades.

Methods Epidemiological data from 1983 to 2007 is based on the cantonal cancer registries and the Federal Statistical Office's (FSO) Cause of Death Statistics. Scientific publications are used to put Switzerland's data into an international context and to discuss risk factors and prevention measures. The Swiss Health Survey of the FSO, conducted in 1997, 2002 and 2007, assesses prevention in Switzerland by two questions: One about the use of sun protection and the second on skin examination frequency.

Results For the period 2003-2007, Switzerland's incidence rate of melanoma was of 22.3 and 20.8 per 100000 inhabitants respectively among men and women. Incidence and mortality trends over the last 25 years are analysed by gender, age, birth cohort and region to identify particularly affected groups. Statistical information about primary prevention shows altogether little alteration in sun protection habits. Secondary prevention (screening) has increased from 2002 to 2007 and shows important variation by educational level.

Conclusion Differences in prevention practice by socio-demographic characteristics indicate that prevention activities have had a variable impact on different population groups. Prevention measures could be more focused. More detailed statistical data on prevention practices would allow a better evaluation of prevention in Switzerland.

\section{P1-376 IMPACT ASSESSMENT OF ICDS FOOD FORTIFICATION IN THE STATE OF UTTAR-PRADESH, INDIA}

doi:10.1136/jech.2011.142976f.68

${ }^{1} \mathrm{R}$ J Yadav, ${ }^{* 2} \mathrm{P}$ Singh, ${ }^{3} \mathrm{~A}$ Nigam, ${ }^{1} \mathrm{~A}$ Pandey, ${ }^{4} \mathrm{D}$ Gulati. ${ }^{1}$ National Institute of Medical Statistics, Indian Council of Medical Research, New Delhi, India; ${ }^{2}$ Ex. Indian Council of Medical Research, New Delhi, India; ${ }^{3}$ ASDS, Lucknow, India; ${ }^{4}$ World Food Programme, New Delhi, India

Objective We planned to assess the impact of fortified food through a community based research study of children aged $12-59$ months in Uttar Pradesh.

Methods We used a quasi experimental design in Kanpur dehat district where fortified food was to be initiated. Sample size calculation required 750 participants. 30 villages (matched for socioeconomic and demographic factors) from each block (intervention, control and benchmark) were selected. From each village 25 children were selected for anthropometrics and dietary intake recording, 10 children for clinical examinations and five for biochemical examinations. Base line assessment was completed in all blocks. The follow-up survey was completed after 1 year of intervention.

Results The majority of children belonged to lower socio-economic groups and underprivileged areas. Considerable improvements in anaemia and serum retinol occurred. The prevalence of severe malnutrition declined considerably in the intervention and control blocks.

Conclusion We found that fortified panjiri was effective in improving anaemia, serum retinol and nutritional status. Short duration of intervention was as effective as prolonged interventions.

\section{P1-377 TRENDS AND INCIDENCE OF AND MORTALITY AFTER FIRST MYOCARDIAL INFARCTION IN A NATIONAL-WIDE COHORT OF MEN AND WOMEN WITH DIVERSE SOCIOECONOMIC POSITION AND COUNTRY OF BIRTH, 1987-2007}

doi:10.1136/jech.2011.142976f.69

D Yang, ${ }^{*}$ D Dzayee, 0 Beiki, L Alfredsson, U de Faire, T Moradi. Karolinska Institutet, Stockholm, Sweden

Introduction Myocardial Infarction (MI) is a major cause of mortality and morbidity worldwide and one of the dominating burden of diseases in Sweden. We evaluated the incidence and survival of first MI in subgroups of social position and sex and compared immigrants with Sweden-born population.

Methods We followed a nation-wide cohort of more than seven million men and women ages 35 to 89 years through linkages between Swedish National Registers from 1987 to 2007. Incidence rate ratio (IRR) was estimated using Poisson regression. Of which, 347834 non-fatal first MI patients were studied for their prognosis using Cox proportional hazard model. 
Results and Conclusion We found immigrants from Southern Asia to Western Asia had higher and immigrants from Eastern and Southerneastern Asia had lower risk of first MI than people born in Sweden. Within Europe, there seemed to be a slightly increasing risk trend from Southern and Western Europe to Eastern and Northern Europe. Low socioeconomic position was independent risk factor of first MI with education as the strongest predictor. However, we found occupation-based index might be more appropriate in immigrants study. Low education was also an independent risk factor for first MI survival. Both first MI risk and mortality decreased over the study period. Another novel finding was the surprisingly protective effect of with history of hyperlipidaemia or hypertension in the prognosis of first MI patients compared to those without.

\section{P1-378 WITHDRAWN}

\section{P1-379 PROJECTING PREVALENCE BY STAGE OF CARE FOR COLON CANCER AND ESTIMATING FUTURE HEALTH SERVICE NEEDS IN NEW SOUTH WALES AUSTRALIA}

doi:10.1136/jech.2011.142976f.70

${ }^{1,2} \mathrm{X} O \mathrm{Yu},{ }^{*}{ }^{3,4} \mathrm{M}$ Clements, ${ }^{1} \mathrm{D}$ O'Connell. ${ }^{1}$ Cancer Council, New South Wales, Sydney, Australia; ${ }^{2}$ The University of Sydney, Sydney, Australia; ${ }^{3}$ Australian National University, Canberra, Australia; ${ }^{4}$ Karolinska Institutet, Stockholm, Sweden

Aim To estimate the number of colon cancer patients in New South Wales (NSW) Australia requiring cancer care in the future.

Methods Prevalence of first primary colon cancer was estimated and projected forward, by stage of clinical care (initial care, long-term survivors, monitoring, and last year of life), for 2007-2016. Then using data from a patterns of care study for colorectal cancer, we estimated the type and amount of treatments that will be needed by colon cancer patients in each stage of care. Finally, data on metachronous tumours or second primary colon cancers were used to estimate the additional number of patients who will require care.

Results In NSW in 2006, there were 17687 patients aged 18-84 years alive with a past diagnosis of colon cancer. By 2016, this number will have increased to 22262 . The expected numbers of patients vary by stage of care with the majority in the monitoring stage $(41.4 \%)$ or being long-term survivors $(40.7 \%)$. There will be at least 1389 men and 1135 women requiring initial surgery for colon cancer in 2016. Of these 431 men and 352 women will also require adjuvant chemotherapy. In addition, there will be 741 patients with metachronous tumours or second primary colon cancers who will also need care in 2016 with $56 \%$ of them in the monitoring stage.

Conclusions These estimates reveal that in NSW the numbers of colon cancer patients requiring care for colon cancer are projected to rise by over $25 \%$ from 2006 to 2016 .

\section{P1-380 SOCIAL NETWORK AND BINGE DRINKING IN SCHOOLS ADOLESCENTS}

doi:10.1136/jech.2011.142976f.71

\author{
${ }^{1,2} \mathrm{P}$ M Zarzar, ${ }^{*} \mathrm{~K} O \mathrm{~J}$ Jorge, ${ }^{1} \mathrm{E}$ Ferreira, ${ }^{1} \mathrm{M} \mathrm{P}$ Vale, ${ }^{2} \mathrm{~T}$ Oksanen, ${ }^{2} \mathrm{~K}$ Kawachi. \\ ${ }^{1}$ Universidade Federal de Minas Gerais, Belo Horizonte, Minas Gerais, Brazil; ${ }^{2}$ Harvard \\ School of Public Health, Boston, Massachusetts, USA
}

Introduction Hazardous alcohol drinking among the adolescent is of major concern. However, it is not known whether the type of social network is associated with binge drinking.

Methods We carried out a cross-sectional study among a random sample of 891 adolescents (40\% male, aged 15-19 years) from public to private schools in 2009-2010 in Belo Horizonte, Minas Gerais,
Brazil. Information on friendship network and binge drinking were collected using two validated self-administered questionnaires: the Integrated Questionnaire for the Measurement of Social Capital (CS-IO) and the Alcohol Use Disorders Identification Test (AUDIT). The Social Vulnerability Index (SVI) was used to assess area-level socioeconomic status. Multinomial regression analysis was used to estimate the associations between the type of most important friends and the frequency of binge drinking.

Results A total of 321 (36\%) adolescents reported binge drinking. We found no differences in binge drinking by age or gender whereas adolescents in private schools, in areas of less vulnerability and higher education of mother reported more binge drinking. Adjusted for age, sex and SVI, reporting friends from school rather than from church as the most important group of friends was associated with a higher likelihood of binge drinking at least monthly (OR 3.1 95\% CI 1.8 to 5.3) and at least weekly (OR $3.295 \%$ CI 1.4 to 7.2 ) when compared to never binge drinking, Lower socioeconomic status in the area was associated with lower likelihood of binge drinking. Conclusions Social network of adolescents is related to binge drinking.

\section{P1-381 THE IMPORTANCE OF SCHOOL-BASED PREVENTION STRATEGIES ON CARDIOVASCULAR HEALTH EDUCATION OF FAMILIES}

doi:10.1136/jech.2011.142976f.72

E Zera, ${ }^{*}$ K Sotirofski, K Leka. Regional hospital, Durres, Albania

Objective The goal of prevention programs is to reduce population exposure to environmental risk factors. Many people do not have sufficient knowledge about cardiovascular risk factors in Durres district.We used a school-based educational intervention program to promote the health knowledge in all participating families. The aim of study is to evaluate the school-based education intervention in improving cardiovascular risk factor knowledge of families.

Methods A total of 400 fifth-grade school children and their parents were invited in the study; 300 families were included in the study. The children attended a single session and were supplied with family information packages. Pre-and post-test questionnaires were completed by these families before and after the session. The questionaire aims to evaluate the knowledge collective not individual. There are questions to risk factor of $\mathrm{CHD}$, to nutrition and their contribute in CHD

Results There was a significant increase in the overall cardiovascular risk factor knowledge of families ( $p$ value $<0.001$ ). The parents of boys achieved a greater level of health knowledge in comparison to the parents of girls ( $p$ value $<0.005$ )

Conclusions This school-based educational intervention was effective in improving cardiovascular risk factor knowledge of families. Similar programs with more comprehensive methodology could be more effective in promoting healthy behaviours.

\section{P1-382 PREVALENCE OF ANAEMIA IN PATIENTS ADMITTED TO HOSPITAL WITH A PRIMARY DIAGNOSIS OF CONGESTIVE HEART FAILURE}

doi:10.1136/jech.2011.142976f.73

E Zera, ${ }^{*}$ A Hatellari. Regional hospital, Durres, Albania

Objectives To find the prevalence of anaemia in patients hospitalised with diagnosis of congestive heart failure (CHF) in our department of cardiology. 